\title{
Adaptive Resource Allocation in MIMO - OFDMA based on Subcarrier Allocation (SA) and (GA)
}

\author{
K. Sumathi \\ Assistant Professor/ECE \\ Dr.Mahalingam College of Engineering and \\ Technology, Pollachi Tamil Nadu, India
}

\author{
M.L Valarmathi \\ Phd,Assistant Professor/CSEGovernment \\ College of TechnologyCoimbatore, Tamil Nadu, \\ India
}

\begin{abstract}
In recent years, the eyes of scholars has been on the road to an proficient allocation scheme of signalling dimension between users in both uplink and downlink channels, since bandwidth is usually limited and/or very expensive. This paper covenants with the rate adaptive MIMO-OFDMA (Multiple Input Multiple Output-Orthogonal Frequency Division Multiplexing) downlink resource allocation scheme. It has an objective of maximizing the total system capacity subject to constraints on total power, bit error rate and proportional fairness. The proposed algorithm determines the number of subcarriers for each user and then handing over the subcarrier to each user. Two optimization methods, one based on Subcarrier Allocation (SA) and the other, based on Genetic Algorithm (GA) are used for resource allocation. In the optimization technique based on SA, using SVD (Singular Value Decomposition), the MIMO fading channel of each subcarrier is transformed into an equivalent bank of parallel SISO (Single Input Single Output) subchannels, which leads to a better trade-off strategy among system capacity, computational complexity and proportional fairness. Paralleled to static allocation schemes, the new algorithm has significantly lower complexity and in simulation fruitages higher system capacity. Simulation results show that the subcarrier allocation based on GA can provide capacity improvement with the reduction in fairness.
\end{abstract}

\section{General Terms}

Wireless Communications, Resource allocation

\section{Keywords}

OFDM, MIMO-OFDMA, resource allocation, Subcarrier Allocation (SA), power allocation, fairness, Genetic Algorithm (GA).

\section{INTRODUCTION}

It is necessary to give attention to the resource allocation as the need for high speed communication increases. In wireless communication the available spectrum is very limited, but the users are more. There comes the effective way allocating the resources namely subcarriers, and subchannels adaptively in a fair and efficient way.

OFDM is a bandwidth efficient technique for multicarrier modulation. High data rates are achieved using OFDM (Orthogonal Frequency Division Multiplexing). In OFDM the wideband channel is partitioned into several narrowband channels by means of IFFT. OFDM divides the available bandwidth into many subchannels that are orthogonal to each other. This principle avoids the interference between the subcarriers. By adding Cyclic Prefix (CP), Inter-symbol Interference (ISI) on the carriers is removed. This makes OFDM suited to wireless communications applications. Some of the applications of OFDM are DAB, DVB. It is an efficient approach to combat the adverse effects of multipath spread, and is the main interface solution to many wireless systems [17] OFDMA (Orthogonal Frequency Division Multiple Access) is an extension of OFDM. OFDMA allows multiple users to transmit simultaneously on different subcarriers per OFDM symbol. A joint bit, power and subcarrier allocation is probed in [14].

The advent of multiple-input multiple-output (MIMO) technology is used in providing high rate reliable wireless communications [21]. MIMO/OFDM systems are able to multiplex the users in both the space and frequency domains. Multiple antennas increase capacity, transmission range reliability and suppress interfering signals. Several studies $[4,8$, 16, and 17] of MIMO/OFDM carried out in multi-user environments. MIMO wireless technology can able to increase the system capacity of a given channel obeying Shannon's law. By increasing the number of receive and transmit antennas the throughput of the channel can be increased linearly with every pair of antennas added. Hence MIMO wireless technology is one of the most important wireless techniques for future communication.

Two classes of optimization techniques are in MIMO-OFDMA. They are margin adaptive (MA) $[4,14,17]$ and rate adaptive (RA) $[3,7,8,9,11,12,13,15,16]$. The objective of margin adaptive is to minimize overall transmit power when users' data rates are given. The rate adaptive objective is to maximize each user's error free capacities with a total transmit power constraint. 
Here we have to allocate the subcarrier and power in fair manner. The power doling out and subcarrier doling out scheme is devised to ensure fairness among users. In the first optimization technique, a value by the name, Fairness Safeguarding Factor (FSF) is introduced in the last part of the proposed subcarrier doling out algorithm to altercate subcarriers among users to ensure fairness. In the second optimization technique, Genetic algorithm (GA) is used to do subcarrier and power doling out.

The rest of this paper is organized as follows. In section 2, the MIMO-OFDMA system model and the capacity optimization objective function are introduced. In section 3 resource doling out based on the two optimization techniques are discussed. Section 4 shows simulation results and conclusions are given in section 5 .

\section{SYSTEM MODEL}

The block diagram of MIMO-OFDMA downlink system model is shown in the Figure 1. It is assumed that perfect channel state information is available at both the base station transmitter and receiver. At the base station, the resource doling out scheme is furthered to the MIMO-OFDM transmitter. This information is also sent via separate channel to the user. The user's data is transmitted via the MIMO channel from the base station and received by the user. The user decodes the data with the help of subcarrier doling out scheme received from the base station via a separate channel.

We assume a total of ' $U$ ' users in the system sharing ' $S$ ' subcarriers with total power $\mathrm{P}_{\text {tot }}$ and BER constraint. Our objective is to maximize the total system capacity and care about fairness among users. Each user has ' $R$ ' receiving antennas and the base station has ' $T$ ' transmitting antennas. The channel gain of user ' $u$ ' on subcarrier ' $s$ ' is denoted as $g_{u, s}$. The total available bandwidth is $\mathrm{B}$ and total power is $\mathrm{P}_{\text {tot }}$. Each subcarrier ' $s$ ' of user ' $u$ ' is assigned a power $p_{u, s} . N_{o}$ is the noise power spectral density. The corresponding signal-to-noise ratio (SNR) is $\left|g_{u, s}\right|^{2} / N_{o} B / S$ and the $\mathrm{u}^{\text {th }}$ users received SNR is given by $\gamma_{\mathrm{u}, \mathrm{s}}=\frac{p_{u, s}\left|g_{u, s}\right|^{2}}{N_{o} B / S}$.

To meet the BER constraints, the effective SNR has to be adjusted accordingly. The BER of a square M-level QAM with Gray-bit mapping as a function of received SNR $\gamma_{\mathrm{u}, \mathrm{s}}$ and number of bits $r_{u, s}$ can be approximated to within $1 \mathrm{~dB}$ for $r_{\mathrm{u}, \mathrm{s}}>4$ and BER $<10^{-3}[18]$.

$$
B E R_{M Q A M}\left(\gamma_{u, s}\right) \approx 0.2 \exp \left[\frac{-1.6 \gamma_{u, s}}{2^{r_{u, s}}-1}\right]
$$

Solving for $r_{\mathrm{u}, \mathrm{s}}$, we get

$$
r_{u, s}=\log _{2}\left(1+\frac{\gamma_{u, s}}{\Gamma}\right)
$$

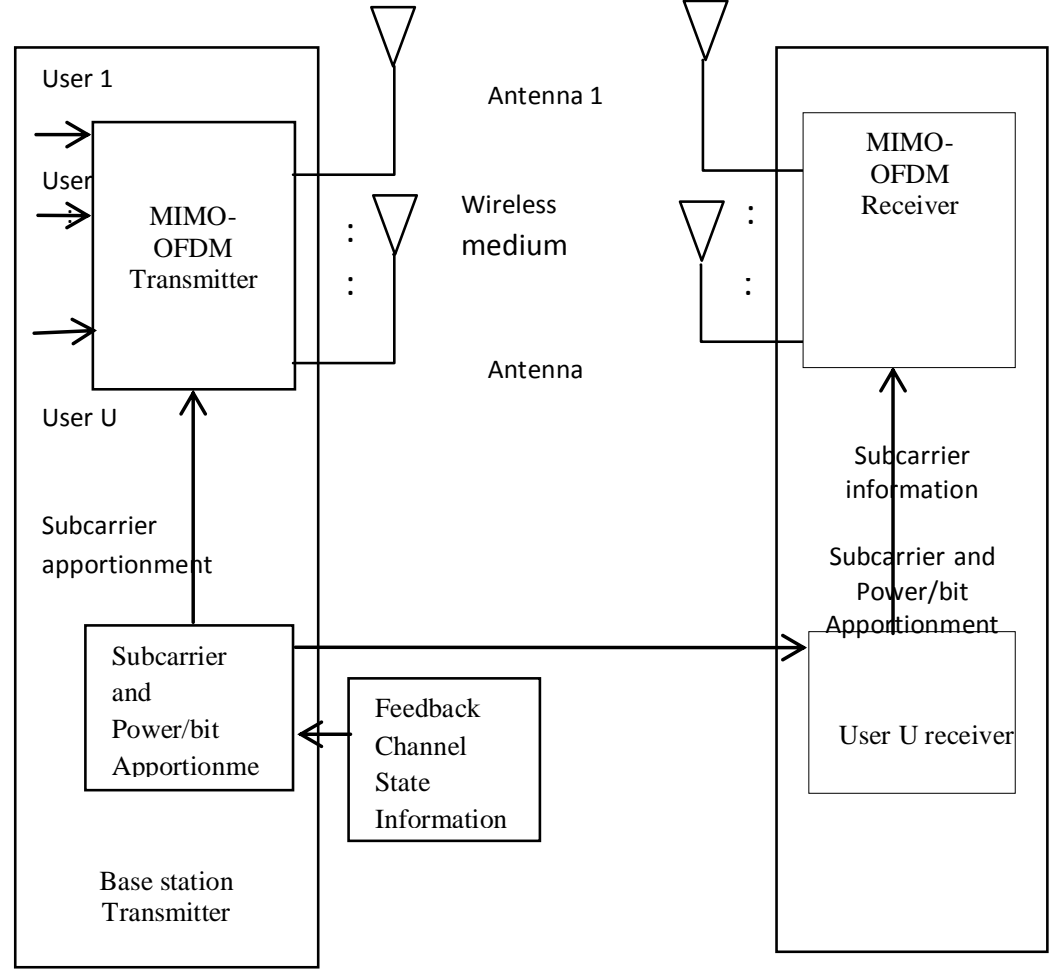

Fig 1. MIMO-OFDMA downlink system model

where $\Gamma=-\ln (5 B E R) / 1.6$, is known as SNR gap. The factor $\Gamma$ which is the difference between the SNR needed to achieve a certain transmission data rate for a practical system and the theoretical limit is called the SNR gap. The total data rate for user ' $u$ ' is

$$
R_{u}=\frac{B}{S} \sum_{s \in \Omega_{u}} r_{u, s}
$$

It is assumed that the subcarriers cannot be shared among users. Each subcarrier has narrowband channel with ' $T$ ' antennas at the base station and ' $R$ ' antennas at the receiver, which can be modelled by an $R \times T$ channel matrix $\mathrm{H}=\left[\mathrm{h}_{\mathrm{ij}}\right]$, where $\mathrm{h}_{\mathrm{ij}}$ is the channel gain at the receive antenna $i$ from transmit antenna $j$. The SVD of $\mathbf{H}$ can be written as

$$
\mathbf{H}=\mathbf{U} \Lambda^{0.5} \mathbf{V}^{*}
$$

Where $\mathbf{U}$ and $\mathbf{V}$ are unitary matrices, $\mathbf{V}^{*}$ denotes the transpose conjugate of $\mathbf{V}$ and $\boldsymbol{\Lambda}$ is a diagonal matrix. The elements of $\boldsymbol{\Lambda}=\operatorname{diag}\left(\left[\tau_{1}, \tau_{2}, \ldots \ldots ., \tau_{M}\right]\right)$ are real and $\mathrm{M}=$ minimum $(\mathrm{T}$, $\mathrm{R})$. Usually, the $\tau_{1}$ value is higher than the other values. For this reason $\tau_{1}$ is called as dominant Eigen mode channel. The SVD operation converts MIMO channel into independent SISO channels, where each channel carriers a portion of users data.

The optimization problem in this paper is probed as 
$\max _{p_{u, s}} \frac{B}{S} \sum_{u=1}^{U} \sum_{s \in \Omega_{u}}\left[\sum_{i=1}^{M} \log _{2}\left(1+\frac{p_{u, s}\left|g_{u, s}^{(i)}\right|^{2}}{N_{o} B / S \Gamma}\right)\right]$

subject to

$C 1: \bigcup_{u=1}^{U} \Omega_{u} \subseteq\{1,2, \ldots, S\}$ with $\left\{\Omega_{u}\right\}_{u=1}^{U}$ being disjoint sets

$\mathrm{C} 2: \sum_{u=1}^{U} \sum_{s \in \Omega_{u}} p_{u, s} \leq P_{t o t}$ with $p_{u, s} \geq 0, \forall u, s$

$C 3: R_{1}: R_{2}: \ldots \ldots: R_{U}=\alpha_{1}: \alpha_{2}: \ldots \ldots: \alpha_{U}$

$\Omega_{\mathrm{u}}$ is the set of subcarriers assigned to user ' $\mathrm{u}$ '. The constraints are denoted by $\mathrm{C} 1-\mathrm{C} 3$. The first constraint is on subcarrier allocation to ensure that each subchannel is assigned to only one user. The next constraint is on power doling out. C3 denotes the rate constraints of the system. The predetermined values $\{\alpha\}_{u=1}^{U}$ are used to ensure proportionality among users.

The capacity of user ' $u$ ' is given by,

$$
R_{u}=\frac{B}{S} \sum_{s \in \Omega_{u}}\left[\sum_{i=1}^{M} \log _{2}\left(1+\frac{p_{u, s}\left|g_{u, s}^{(i)}\right|^{2}}{N_{o} B / S \Gamma}\right)\right]
$$

The optimization problem given in (5) is NP-hard in nature, which means that an acceptable solution cannot be obtained with low complexity and in a short span of time. To solve this issue, two optimization techniques are proposed in the next section.

\section{NEW SUBCARRIER DOLING OUT SCHEME}

Two optimization methods are explained here. First one is optimization based on SA followed by optimization based on GA.

\subsection{Optimization Based On Subcarrier Allocation (SA)}

In this sliver, we discuss a suboptimal subcarrier doling out algorithm. The algorithm is suboptimal because equal power distribution in all subcarriers is assumed [19]. To reduce complexity, this scheme consists of two separate stages which are subcarrier doling out and power doling out as in $[4,16$, and 17].

Step 1: Determine the number of subcarriers to be initially assigned to each user and unused subcarriers.

Step 2: Assign the subcarriers to each user as determined in the previous step. Then assign the unused subcarriers (if any) to the users with the aim of enhancing total system capacity.

Step 3: A Fairness Safeguarding factor (FSF) is used to reorganize subcarriers among users to ensure fairness.

Various notations used in the algorithm is explained below. $\mathrm{c}_{\mathrm{u}, \mathrm{s}}$ is the subcarrier doling out indicator and $c_{u, s}=1$, if subcarrier ' $\mathrm{s}$ ' is assigned to user ' $\mathrm{u}$ '. $\mathrm{S}_{\mathrm{u}}$ denotes number of subcarriers needed for user ' $u$ '. $S_{\text {unused }}$ is the number of unassigned subcarriers. $R_{\text {tot }}$ is the total data rate of all users.
In step 1, we determine the number of subcarriers for each user and initialize the variables. This initial step is based on the assumption that the proportion of subcarriers assigned to each user is approximately same as their eventual data rates and would roughly satisfy the proportionality constraints.

Step 2 consists of three substeps as explained below.

In step 2a, each user is assigned the subcarrier having maximum gain for that user. For each user, first subcarrier is allocated under the scheduling principle that the user with less preassigned $S_{u}$ has higher priority to choose his first subcarrier. After doing this step, U subcarriers have been chosen. Thus there are $(\mathrm{S}-\mathrm{U})$ subcarriers available for users to select and user index should be reinitialized in the next step.

In step $2 b$, subcarriers are assigned to user according to the policy that the user with the least ratio of instantaneously achieved rate to its required proportion has the priority to choose one subcarrier at a time.

The user with most starvation to its desired proportion can feed him as much as possible through choosing additional subcarriers with better channel conditions. Since importance is given to proportional rates, the need of a user is determined by the user who has the least capacity divided by its proportionality constant. Once the user gets his allotment of $S_{u}$ subcarriers, he can no longer be assigned any more subcarriers in this step. Proportional fairness among users is emphasized in this step.

In step 2c, remaining subcarriers $S_{\text {unused }}$ are allocated to the best users, wherein each user can get at most one unassigned subcarrier.

In step 3, we reorganize the subcarriers among user with most fairness and least capacity loss. For this reorganization purpose a factor called Deviation Indicator (DI) is introduced. This factor shows the overall deviation of all users from their desired proportions. When this value becomes smaller, the proportional fairness would be enhanced. $\mathrm{DI}=0$ indicates ideal fairness.

The DI is given by

$$
D I=\left(\frac{1}{U} \sum_{u=1}^{U}\left(\frac{R_{u}}{R_{t o t}}-\beta_{u}\right)^{2}\right)^{\frac{1}{2}}, \beta_{u}=\frac{\alpha_{u}}{\sum_{u=1}^{U} \alpha_{u}}
$$

A Fairness Safeguarding Factor (FSF) is used to control the number of subcarrier altercations. In each loop, subcarriers between two selected users with most unfair proportions are altercated. Thus this step is an iterative altercation process, which can enhance fairness at the cost of losing certain amount of capacity. The steps of the proposed algorithm are given in Fig 2.

To evaluate the fairness of the algorithm we define a parameter called Fairness Index (FI) as in [20].

The Fairness Index (FI) is defined as, 


$$
F I=\frac{\sum_{u=1}^{U} \alpha_{u}{ }^{2}}{U \sum_{u=1}^{U} \alpha_{u}{ }^{2}}
$$

with the maximum value of 1 to be the fairest case in which all users would achieve the same data rate. Based on the above equation, a new parameter $F_{p}$ is defined to examine the performance of the algorithm in maintaining proportionality which is given by

$$
F_{p}=\frac{\sum_{u=1}^{U} R_{u} / \alpha_{u}{ }^{2}}{U \sum_{u=1}^{U} R_{u} / \alpha_{u}{ }^{2}}
$$

where $R_{u}$ and $\alpha_{u}$ are the achieved rate and the proportional rate constraint for the $u^{\text {th }}$ user respectively. $\mathrm{F}_{\mathrm{p}}$ is a real number in the interval $(0,1]$ with the maximum value of 1 for the case that the achieved rate proportions among the users are the same as the predetermined set $\left\{\alpha_{u}\right\}_{u=1}^{U}$.

\subsection{Optimization Based On Genetic Algorithm (GA)}

Genetic algorithm (GA) is a search heuristic that is derived from natural evolution. This heuristic is commonly used to generate useful solutions to optimization and search problems. Genetic algorithms belong to the larger class of evolutionary algorithms (EA), which generate solutions to optimization problems using techniques inspired by natural evolution, such as inheritance, mutation, selection, and crossover.

It is a method for solving both constrained and unconstrained optimization problems that is based on natural selection, the process that drives biological evolution and is based on the nature of survival of the fittest. Genetic Algorithms (GA) are not too hard to program or understand since they are biological based. Genetic algorithms are acknowledged as good solvers for tough problems.

The steps involved in GA are given as flowchart in Fig 3. The algorithm starts with random set of solutions called population.
In each step (generation) new population is achieved from the old one. New individuals are created by crossing old ones (parents). Based on the fitness values the individual can become a parent.

The mutation is performed after cross over which produce children for next generation. The best solution in current generation is called elite. The population of 30 individuals is considered here for implementing this allocation.

Let an OFDM system have $\mathrm{U}(\mathrm{u}=1,2 \ldots \mathrm{U})$ Users and $\mathrm{S}(\mathrm{s}=1$, 2 .., S) subcarriers. The system assigns a subset of $\mathrm{N}$ subcarriers to a user and determines the number of bits/symbol per each assigned subcarrier on downlink transmission.

1. Initialization: Generate a population Npop of chromosomes, where each bit of all the chromosomes is randomly picked from 1 to $\mathrm{U}$.

2. Fitness evaluation: Update power allocation for each chromosome by using the subcarrier allocation presented by the chromosome and utilizing the corresponding power and subcarrier allocation of each chromosome, obtain the fitness value of each chromosome, which is the weighted capacity.

3. Elitism: Find Nelite chromosomes with the highest fitness values; copy them into the next generation directly.

4. Crossover: Pick two chromosome parents from the current generation to create chromosome children for the next generation. Randomly obtain a crossover point for the parents. Using those parents, single point crossover is done. Similarly, generate Ncross crossover children chromosomes for the next generation.

5. Mutation: Randomly pick a chromosome from the current generation, each bit of it can be changed with a chance of Pmu. Generate Nmu mutation children chromosomes for the next generation.

6. Repeat Steps 2, 3, 4 and 5 until reaching the maximum generation limit Ngen. 


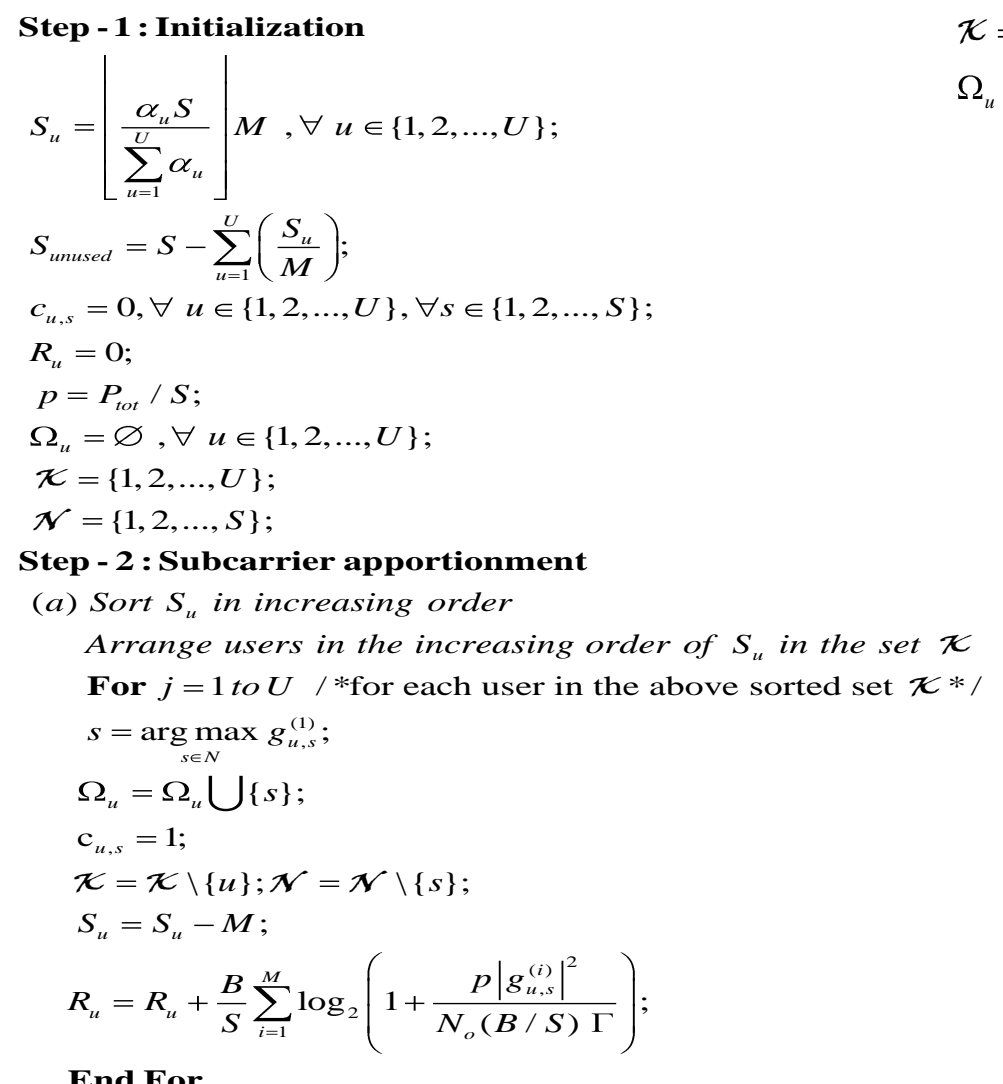

\section{End For}

(b) $\pi=\{1,2, \ldots, U\}$;

While $\|\boldsymbol{N}\|>S_{\text {unused }}$ Do

$$
\begin{aligned}
& \beta_{u}=\frac{\alpha_{u}}{\sum_{u=1}^{U} \alpha_{u}} \\
& \mathrm{u}=\underset{u \in \mathcal{K}}{\arg \min }\left(R_{u} / \beta_{u}\right) ; \\
& \mathbf{I f} \mathrm{S}_{u}>0 \text { Do } \\
& \mathrm{s}=\underset{s \in \mathcal{N}}{\arg \max } g_{u, s}^{(1)} ; \\
& \Omega_{u}=\Omega_{u} \bigcup\{s\} ; \\
& \mathrm{C}_{u, s}=1 ; \\
& \mathbb{N}=\mathbb{N} /\{s\} ; \\
& \mathrm{S}_{u}=S_{u}-M ; \\
& R_{u}=R_{u}+\frac{B}{S} \sum_{i=1}^{M} \log _{2}\left(1+\frac{p\left|g_{u, s}^{(i)}\right|^{2}}{N_{o}(B / S) \Gamma}\right) ;
\end{aligned}
$$$$
\mathcal{K}=\mathcal{K} \backslash\{u\} ;
$$$$
\Omega_{u}=\Omega_{u} \bigcup\{s\} \text {; }
$$$$
c_{k, n}=1
$$$$
\mathcal{N}=\mathcal{N} /\{s\}
$$$$
R_{u}=R_{u}+\frac{B}{S} \sum_{i=1}^{M} \log _{2}\left(1+\frac{p\left|g_{u, s}^{(i)}\right|^{2}}{N_{o}(B / S) \Gamma}\right)
$$

\section{End For}

\section{Step - 3 : Subcarrier reorganization}

While $F S F>0$ Do

$$
\begin{aligned}
& \mathcal{K}=\{1,2, \ldots, \mathrm{U}\} \text {; } \\
& \mathcal{N}=\{1,2 \ldots, \mathrm{S}\} \text {; } \\
& R_{\text {tot }}=\sum_{u=1}^{U} R_{u} \\
& \mathrm{a}=\underset{a \in \mathcal{K}}{\arg \min }\left(R_{a} / R_{t o t}-\beta_{a}\right) ; \\
& b=\underset{b \in \mathcal{K}}{\arg \max }\left(R_{b} / R_{t o t}-\beta_{b}\right) \text {; } \\
& D I^{\text {pre }}=\left(\frac{1}{U} \sum_{u=1}^{U}\left(R_{u} / R_{t o t}-\beta_{u}\right)^{2}\right)^{1 / 2} ; \\
& R_{t 1}=R_{a} \text {; } \\
& R_{t 2}=R_{b} \\
& e=\underset{s \in \mathcal{N}}{\arg \min }\left(c_{b, s} g_{b, s}^{(1)}-c_{a, s} g_{a, s}^{(1)}\right)^{2} \text { for } e \neq 0 ; \\
& R_{a}=R_{a}+\frac{B}{S} \sum_{i=1}^{M} \log _{2}\left(1+\frac{p\left|g_{a, e}^{(i)}\right|^{2}}{N_{o}(B / S) \Gamma}\right) \\
& R_{b}=R_{b}-\frac{B}{S} \sum_{i=1}^{M} \log _{2}\left(1+\frac{p\left|g_{b, e}^{(i)}\right|^{2}}{N_{o}(B / S) \Gamma}\right) \\
& D I^{p o s t}=\left(\frac{1}{U} \sum_{u=1}^{U}\left(R_{u} / R_{t o t}-\beta_{u}\right)^{2}\right)^{1 / 2} ; \\
& \text { If } \quad D I^{\text {post }}<D I^{\text {pre }} \text { Do } \\
& c_{b, e}=0 \text {; } \\
& c_{a, e}=1 ;
\end{aligned}
$$

Else $R_{s}=R_{t 1} ; R_{a}=R_{t 2}$;

break;

$F S F=F S F-1 ;$

End If

End While

Fig 2. Steps in the algorithm based on Subcarrier Altercation (SA) 


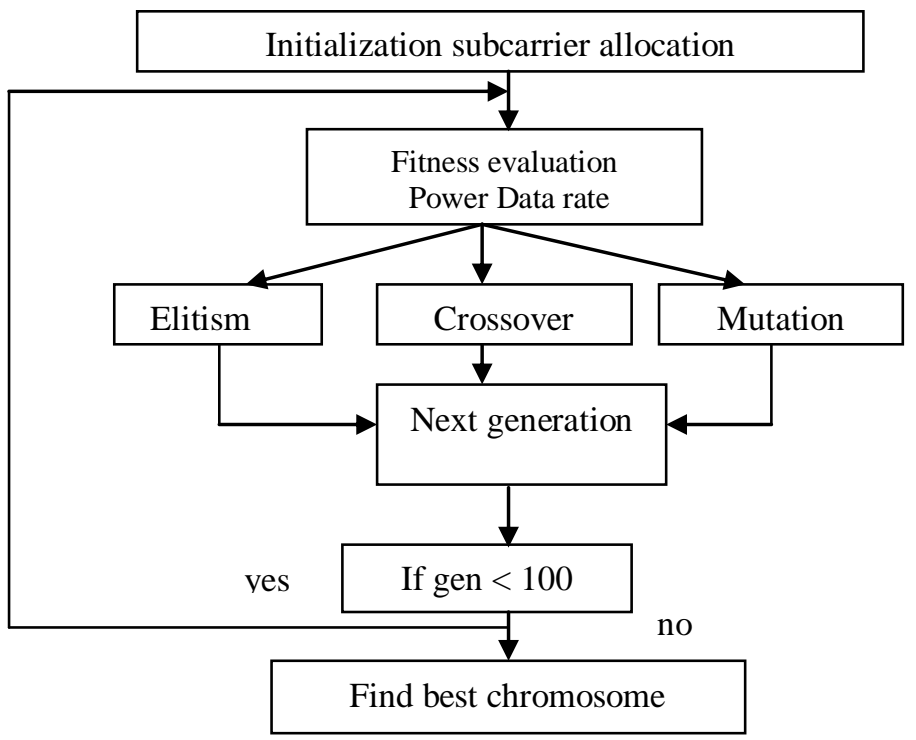

Fig 3. Steps in Genetic Algorithm (GA)

\section{SIMULATION RESULTS}

Here in the first part the simulation parameters required for doing the simulation is dealt followed by simulation snapshots and its descriptions

\subsection{Simulation Parameters}

The simulation is done with the following parameters as shown in Table 1. Here we have taken the number of users as 8 and the subcarriers are 128 considering the bandwidth as $1 \mathrm{MHz}$.

Table 1. Simulation Parameters

\begin{tabular}{|c|c|}
\hline PARAMETER & VALUE \\
\hline $\mathrm{U}$ & 8 \\
\hline $\mathrm{S}$ & 128 \\
\hline B & $1 \mathrm{MHz}$ \\
\hline $\mathrm{P}_{\text {tot }}$ & $1 \mathrm{~W}$ \\
\hline BER & $10^{-3}$ \\
\hline Doppler shift & $30 \mathrm{~Hz}$ \\
\hline $\begin{array}{c}\text { Maximum delay } \\
\text { spread }\end{array}$ & $5 \mu \mathrm{s}$ \\
\hline $\mathrm{N}_{\mathrm{o}}$ & $-80 \mathrm{dBW} / \mathrm{Hz}$ \\
\hline Channel model & $\begin{array}{l}\text { Frequency selective Rayleigh channel with } \\
\text { six multipath }\end{array}$ \\
\hline FSF & $0,5,10,24$ \\
\hline $\mathrm{T}=\mathrm{R}$ & 2 \\
\hline $\begin{array}{l}\text { Proportionality } \\
\text { constants, } \alpha\end{array}$ & $p_{\alpha}=\left[\begin{array}{l}1 \text { with probability } 0.5 \\
2 \text { with probability } 0.3 \\
4 \text { with probability } 0.2\end{array}\right]$ \\
\hline $\mathrm{N}_{\text {pop }}$ & 30 \\
\hline $\mathrm{N}_{\text {elite }}$ & 10 \\
\hline $\mathrm{N}_{\text {cross }}$ & 14 \\
\hline $\mathrm{N}_{\mathrm{mu}}$ & 6 \\
\hline $\mathrm{P}_{\mathrm{mu}}$ & 0.01 \\
\hline $\mathrm{N}_{\text {gen }}$ & 100 \\
\hline
\end{tabular}

\subsection{Simulation snapshots and descriptions}

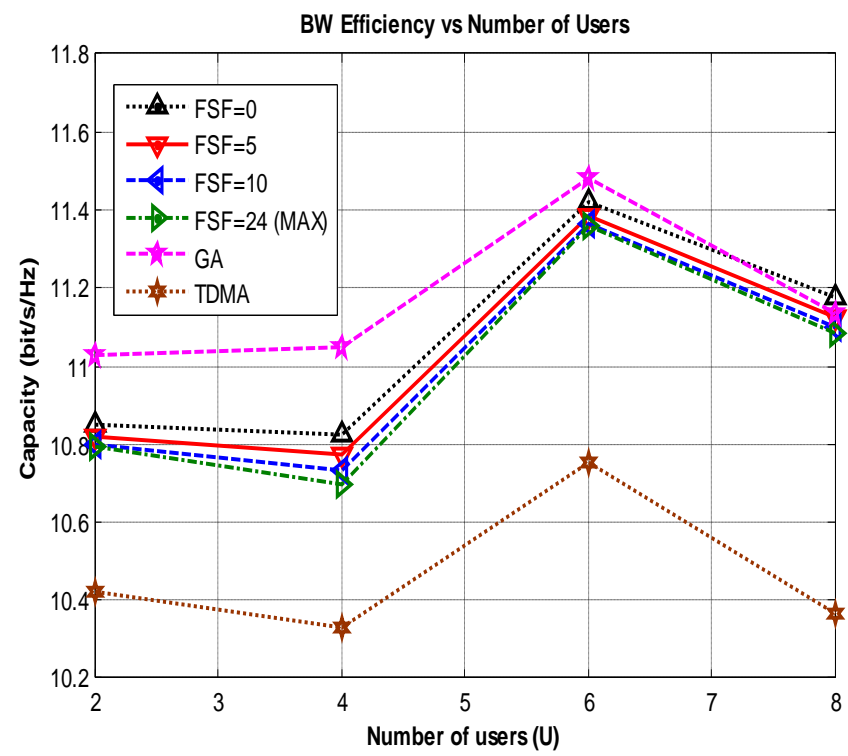

Fig 4. Capacity Vs Number of Users

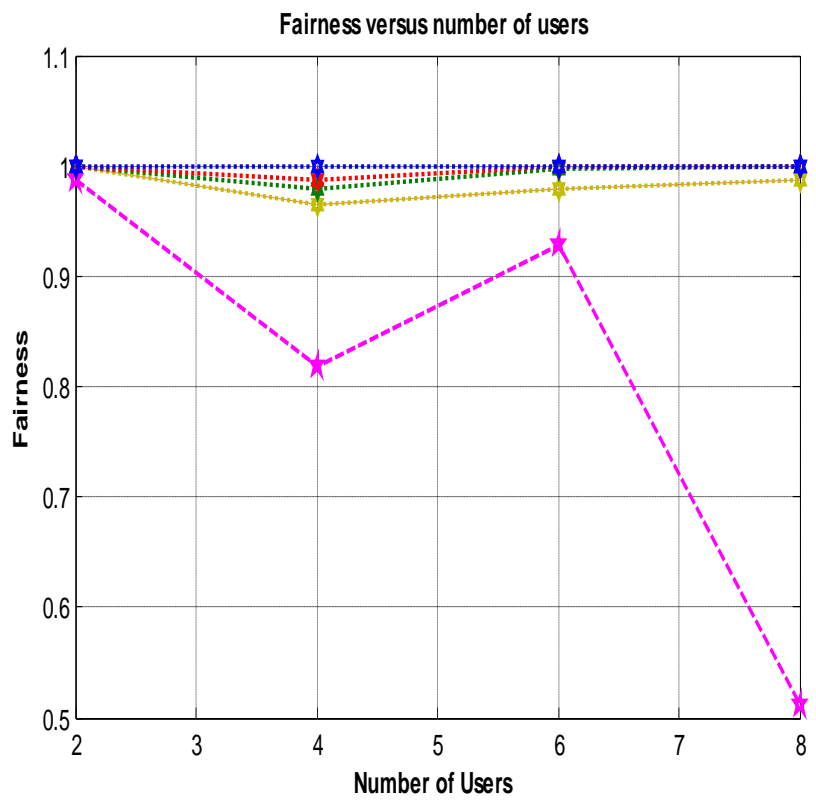

Fig 5. Fairness Vs Number of Users 


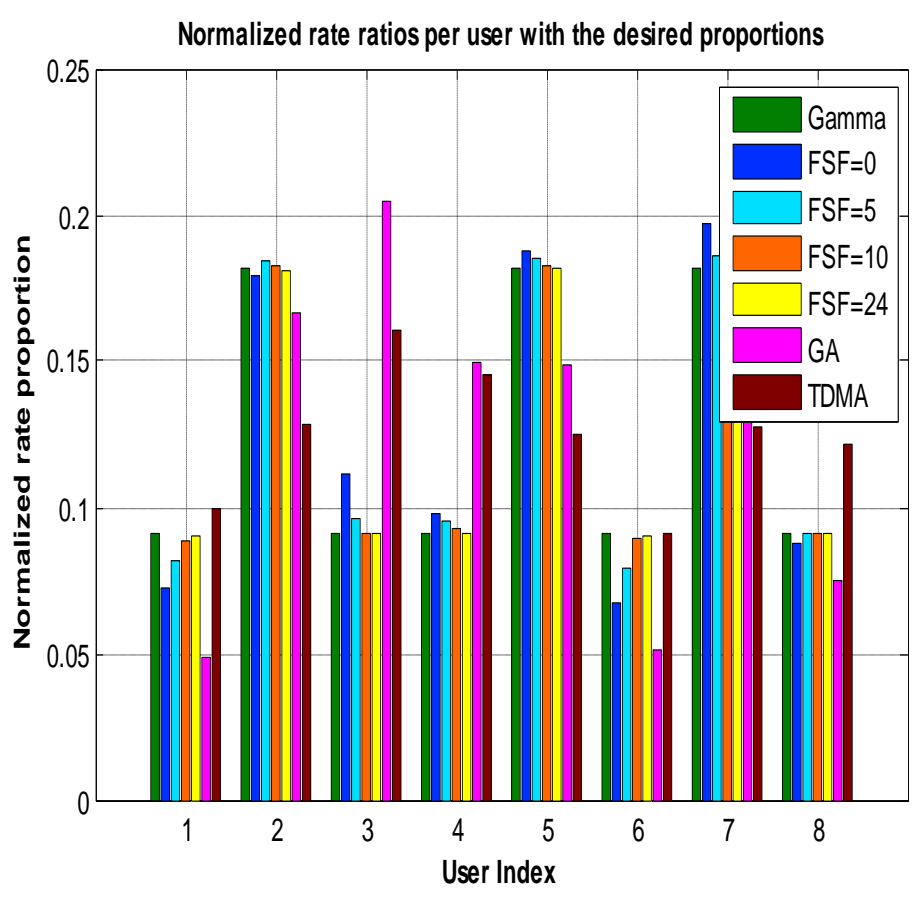

Fig 6: Channel Gain Vs User index

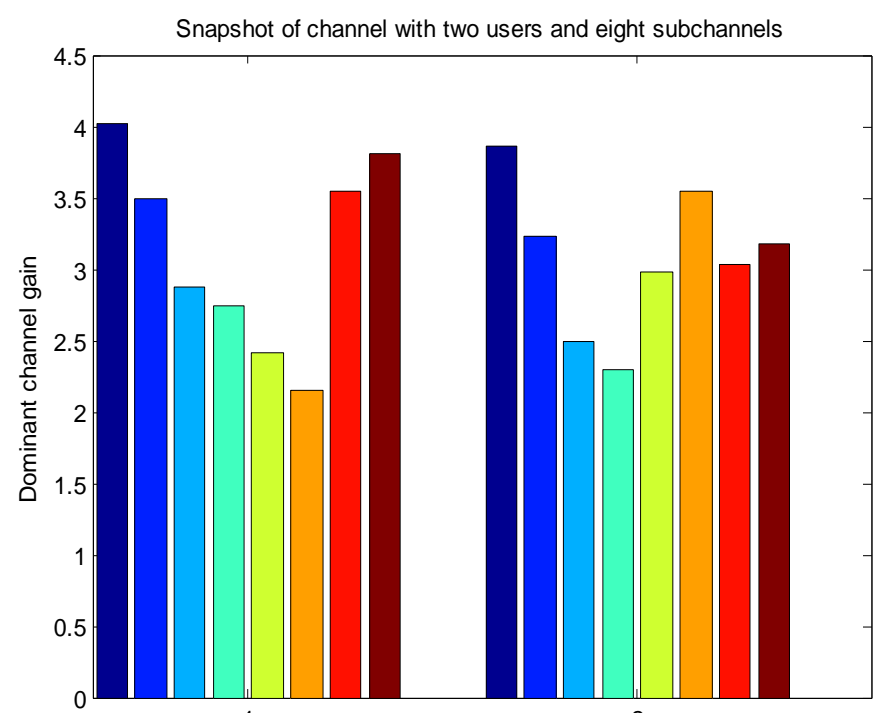

Fig 7. Channel Gain Vs User indêx User index (u)

Fig 4 shows the capacity to number of users. Here numbers of users taken are 8. This figure shows that there is an improvement in capacity when GA is used. There is reduction in capacity when the FSF factor gets increased. When $\mathrm{FSF}=0$ the capacity starts from $10.8 \mathrm{bits} / \mathrm{Hz}$ and reaches a maximum value as 11.4 bits/Hz. For FSF $=5,10,24$ it approximately starts from less than $10.8 \mathrm{bits} / \mathrm{Hz}$ and reaches a maximum value less than 11.4 bits/Hz. For TDMA it starts from 10.8 bits/Hz and reaches a maximum value nearer to $10.8 \mathrm{bits} / \mathrm{Hz}$. Hence from the graph the performance of TDMA scheme is low when compared to SA and GA.
Fig 5 shows relation between fairness and the number of users. Here compared to the other methods GA provides increased capacity but at the cost of reduction in fairness among users. If fairness is not a main criterion then for capacity improvement GA can be used. GA provides poor fairness if the users starts increasing.

Fig 6 shows normalized rate proportion to user index. The normalized proportions for 8 users were taken. Here it was shown that the proposed scheme with maximum FSF value can attain close to optimal fairness. The maximum FSF value is chosen as 24 for the case of system with 8 users. The normalized capacities are given by $R_{u} / \sum_{k=1}^{16} R_{u}$. This is compared to the normalized proportionality constants. The rate is proportionally assigned to the users. When $\mathrm{FSF}=24$, gives moderate performance. The performance of GA is also considered to be good over other methods.

Fig 7 shows channel gain to user index. Channel characteristics for a system with two users and eight subchannels. The channel gains of user 1 have large variance while that of user 2 have smaller variance. It is due to multiuser diversity that a subchannel is rarely in deep fade for all the users. As a result of frequency selectivity of the channel, different subchannels of the same user experience different levels of fade. This figure represents the fact that the same subchannel may look deeply faded to one user while it may look good for the other.

For the GA based subcarrier allocation method, the parameters like the population size is Npop $=30$, consisting of Nelite $=10$ elites, Ncross $=14$ crossover children and $\mathrm{Nmu}=6$ mutation Ngen $=100$ were considered and the mutation probability Pmu is taken as 0:01.

\section{CONCLUSIONS}

In this paper two new resource doling out schemes with flexible controllability on capacity, fairness and complexity has been investigated. This paper gifts a new method to solve the rate adaptive resource doling out problem with proportional rate constraints for the MIMO-OFDMA systems. Under assumption that the perfect channel state information is available at both the transmitter and the receiver, the proposed algorithm presents an in effect scheme to conglomerate the advantages of MIMO, OFDM in the downlink. The proposed scheme based on SL with maximum FSF value achieves almost ideal fairness with a small capacity loss. This new scheme without subcarrier altercation may be used to capacity-emphasized scenario with acceptable reduced fairness. The scheme based on GA achieves higher capacity. But increased capacity with the reduction in fairness among users. So, when higher capacity is the only ultimate one could opt for optimization based on GA. Simulation results show that the proposed algorithms has better performance than the fixed subcarrier doling out scheme and can reduce computational complexity by using the dominant eigen channels (for SA). Using the wished-for schemes the system capacity is distributed more fairly among users. Therefore for different proportionality constraints, different rates can be achieved among users. 


\section{REFERENCES}

[1] Andrea Goldsmith, Wireless Communication, Cambridge University press 2005

[2] G.Bauch and A. Naguib, "Map equalization of space-time coded signals over frequency selective channels", Proc. IEEE Wireless Communications. Network Conf., pp.261265, September, 1999.

[3] Bin Da, C. C. Ko, "A New Scheme with Controllable Capacity and Fairness for OFDMA Downlink Resource Allocation". in Proc. IEEE VTC 2007-fall, pp. 1817-1821.

[4] J. M. Choi, J.S. Kwak, H. S. Kim, and J. H. Lee, "Adaptive Subcarrier Bit, and Power Allocation Algorithm for MIMO-OFDMA System”, in Proc. VTC 2004-Spring, vol.3, pp.1801-1805.

[5] David Tse, Pramod Viswanath, "Fundamentals of Wireless Communication", Cambridge University press 2006.

[6] C. Fragouli, N. A;-Dhahir, and S. Diggavi, "Pre-filtered space-time M-BCJR equalizer for frequency selective channels”, IEEE Trans. Commun., pp. 742-53, May 2002.

[7] J. Hui, and Y. Zhou, "Enhanced Rate Adaptive Resource Allocation Scheme in Downlink OFDMA System", in Proc. IEEE VTC2006-Spring, vol. 5, pp. 2464-2468.

[8] M. Kobayashi, G. Caire, "Iterative Water filling for Weighted Rate Sum Maximization in MIMO OFDM Broadcast Channels", in Proc. ICASSP Conf., vol. 3, April 2007, pp. 5-8.

[9] G. Li, and H. Liu, "On the Optimality of DownlinkOFDMA MIMO Systems", in Proc. Signals, Systems and Computers Conf., vol. 1, November 2004, pp. 324-328.

[10] Naugib, "Equalization of transmitter diversity space-time coded signals,", Proc. IEEE Globecom Conf., December 2000, pp.1077-82.

[11] M.S. Maw, and I. Sasase, "Resource Allocation Scheme in MIMO -OFDMA System for users Different Data Throughput Requirements", in Proc. IEEE WCNC, March 2007, pp. 1706-1710.

[12] W. Rhee, and J. M. Cioffi, "Increase in Capacity of Multiuser OFDM System Using Dynamic Subchannel
Allocation", in Proc. IEEE VTC, Japan, May 2000, pp. 1085-1089.

[13] Z. Shen, J. G. Andrews, and B. L. Evans, "Adaptive Resource Allocation in Multiuser OFDM Systems with Proportional Rate Constraints", IEEE Trans. wireless communication, vol. 4, pp. 2726 - 2737, November 2005.

[14] C. Y. Wong, R. S. Cheng, K. B. Letaief, and R. D. Murch, 'Multiuser OFDM with Adaptive Subcarrier, Bit, and Power Allocation', IEEE J. Select. Areas Communication, vol. 17, pp. 1747- 1758, October 1999.

[15] I. C. Wong, Zukang Shen, B. L. Evans, and J. G. Andrews, 'A Low Complexity algorithm for Proportional Resource Allocation in OFDMA Systems', in Proc. Signal Processing Systems Conf., SIPS 2004, pp. 1-6.

[16] J. Xu, J. Kim, W. Paik, J. S. Seo, 'Adaptive Resource Allocation Algorithm with Fairness for MIMO-OFDMA System', in Proc.VTC 2006-spring, vol. 4, pp. 1585-1589.

[17] Y. J. Zhang, and K. B. Letaief, 'An Efficient ResourceAllocation Scheme for Spatial Multiuser Access in MIMO/OFDM Systems', IEEE Trans Commun., vol.53, pp.107-116, January 2005

[18] S.T. Chung and A.Goldsmith, "Degrees of Freedom in Adaptive Modulation: A Unified View" IEEE Trans. Commun., vol 49, no.9, pp.1561- 1571, September 2001.

[19] Bin Da and C.C. Ko, "Resource Allocation in Downlink MIMO-OFDMA with Proportional Fairness", Journal of Communications, vol.4, no.1, February 2009.

[20] Sanam Sadr, Alagan Anpalagan and Kaamran Raahemifar, "Suboptimal Rate Adaptive Resource Allocation for Downlink OFDMA Systems", International Journal of Vehicular Technology, 2009.

[21] J.Paulraj, D.A.GORE, R.U.Nabar, and H. Bolcskei, “An overview of MIMO communications-a key to gigabit wireless ," Proc. IEEE vol.92,No.2,pp.198-218,2004. 